Karina Batthyány

Universidad de la República, Montevideo, Uruguay

Natalia Genta

Universidad de la República, Montevideo, Uruguay

Valentina Perrotta

Universidad de la República, Montevideo, Uruguay

\title{
El aporte de las familias y las mujeres a los cuidados no remunerados en salud en Uruguay
}

\begin{abstract}
Resumen: Considerando el creciente protagonismo de los cuidados en la agenda pública y de investigación en Uruguay, el presente artículo se enfoca en un tipo de cuidado prestado en forma no remunerada que requiere mayor visualización: el cuidado no remunerado en salud. Se propone responder a las siguientes interrogantes: ¿Quiénes asumen los costos del cuidado no remunerado en salud en Uruguay?, ¿Qué perfil tienen las personas cuidadoras no remuneradas en salud?, ¿A quiénes cuidan? ¿Cuál es el vínculo que mantienen con el sistema institucional de salud? Para ello, utiliza información proveniente de la Encuesta Nacional de Cuidados No Remunerados en Salud, realizada por el Grupo de Investigación Sociología de Género, de la cual las autoras son integrantes. Dicha Encuesta es representativa de la población uruguaya, está basada en una muestra bietápica y estratificada por nivel socioeconómico en la que se consultó a 1198 hogares sobre la realización de cuidados no remunerados en salud. Se demuestra que el cuidado en salud es mayormente realizado por integrantes del hogar, $y$ de forma no remunerada. Las mujeres son las principales cuidadoras en salud, lo que da cuenta de la división sexual del trabajo existente en estas tareas. Por su parte, son las mujeres de mayor edad las que se dedican principalmente a proporcionar este cuidado, y esto es un rasgo distintivo de los cuidados en salud que los diferencia de los cuidados no remunerados cotidianos. Palabras claves: Género, cuidados, salud, uso del tiempo, trabajo no remunerado
\end{abstract}

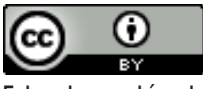

Esta obra está sob licença Creative Commons.

\section{Introducción}

Uruguay se enfrenta al desafío de asumir un nuevo enfoque de las políticas sociales de nueva generación, consistente en el reconocimiento por parte del Estado del 
${ }^{1}$ El Sistema Nacional de Cuidados se propone pasar de un modelo familista a uno de corresponsabilidad social entre varones y mujeres y entre Estado, mercado familias y comunidad, generando cambios en la actual división sexual del trabajo, a través de distintos mecanismos institucionales de regulación y oferta de servicios. Al mismo tiempo plantea la necesidad de valorizar la tarea de cuidados, profesionalizándola a través de la capacitación de las y los cuidadores. Para cumplir con sus objetivos, el Sistema propone la creación de nuevos servicios la regulación de los existentes y la formación de quienes trabajan en los cuidados, así como la promoción activa de las transformaciones culturales hacia la corres ponsabilidad en los cuidados. (CONSEJO NACIONAL DE POLÍTICA SOCIAL, 2012) cuidado como derecho universal. El país discute la implementación del Sistema Nacional Integrado de Cuidados, colocándose como uno de los pioneros en la región en la consideración de los cuidados como un problema público y asumiendo el Estado un rol clave. ${ }^{1}$

Numerosos estudios han demostrado que la generación de bienestar de nuestras sociedades depende en buena medida de las actividades domésticas, en su inmensa mayoría no remuneradas, que son desarrolladas por las familias. El cuidado de la salud constituye una de las actividades no remuneradas con mayor incidencia sobre la vida económica y social de los países. Estudios internacionales (María del Mar GARCÍA- CALVENTE, et al.; 1999; Ximena DÍAZ, et al., 2006; María de los Ángeles DURÁN, 2008) evidencian que el cuidado de la salud de personas dependientes es realizado mayoritariamente por las familias, mientras que las instituciones públicas y privadas participan de forma minoritaria.

La distribución entre Estado, familias, mercado y comunidad de los costos, roles y responsabilidades en la atención de las personas dependientes (niños/as, personas mayores, discapacitados) es lo que se pone en juego en la definición del cuidado como política pública. La intervención y articulación de esos diferentes actores impactan en la posición de las mujeres en las familias y en el mercado de trabajo, así como determinan la efectiva capacidad de las mujeres de ejercer los derechos vinculados a su ciudadanía social.

La provisión de cuidados en salud por parte de las familias carece de visibilidad dadas sus características de trabajo no remunerado. Parte de esta invisibilidad se sustenta en que estos servicios no suponen una retribución monetaria, o una contabilización de los tiempos invertidos, lo que torna difícil su cuantificación y la estimación de su aporte sobre el bienestar social. Ello deriva en que la atención y los cuidados de la salud sean generalmente entendidos y analizados únicamente en el marco del sistema institucional de salud. Dicho sistema conjuga instituciones públicas y privadas para ofrecer servicios profesionales e implica trabajo remunerado del personal de la salud y de los/as cuidadores/as. Sin embargo, estos servicios constituyen sólo la punta de un iceberg en el que el cuidado no remunerado, sobre todo dentro de las familias, se configura como un verdadero sistema invisible de atención a la salud (DURÁN, 2008, p.114).

Las oportunidades actuales con que cuentan las familias para sostener la provisión de cuidados se ven actualmente comprometidas por la denominada "crisis del cuidado". La crisis del cuidado consiste en un desajuste entre la demanda de cuidados, compuesta por las personas dependientes (debido a edad o a enfermedad permanente 
2 Referimos a la definición propuesta por Berenice FISHER y Joan TRONTO (1990) "El cuidado es una actividad específica que incluye todo lo que hacemos para mantener, continuar y reparar nuestro mundo, de manera que podamos vivir en él tan bien como sea posible. Ese mundo incluye nuestros cuerpos, nuestro ser y nuestro ambiente, todo lo que buscamos para entretejer una compleja red del sostenimiento de la vida. (FISHER Y TRONTO, 1990 en Sonia MONTAÑO, 2010, p. 27) o temporal) y la oferta de cuidados, proveniente de las personas disponibles para cuidar. En el contexto de crisis, la oferta de cuidados disminuye mientras que aumenta la demanda. Este desajuste se vincula a una serie de cambios culturales y demográficos entre los que se destacan el aumento de la esperanza de vida, la cual incrementa la demanda de cuidados de personas mayores, la disminución de hogares de tipo tradicional (mujer ama de casa y varón proveedor) y el aumento de la participación de las mujeres en el mercado laboral, que impactan en la disminución de personas, particularmente mujeres disponibles para brindar cuidado.

La constatación del aumento de las necesidades específicas de cuidados en salud es producto de varios factores, entre los que podemos destacar el proceso de envejecimiento, que genera demanda creciente de cuidado a personas mayores, pero también de la vulnerabilidad del sistema sanitario, el que está apoyado en las mujeres y en un modelo familiar biparental tradicional. Dichas causas, entre otras, han derivado en una serie de cuestionamientos. ¿Quiénes asumen los costos del cuidado no remunerado en salud en Uruguay? ¿Qué perfil tienen las personas cuidadoras no remuneradas en salud?, ¿A quiénes cuidan? ¿Cuál es el vínculo que mantienen con el sistema institucional de salud?

\section{Cuidados, género y uso del tiempo}

La noción de cuidados continúa siendo discutida conceptualmente mientras persisten innumerables trabajos de investigación que abordan uno o varios aspectos empíricos relacionados a las actividades de cuidado. Hoy conviven diversas conceptualizaciones acerca del cuidado y las formas de abordarlo (Carol THOMAS, 201 1, p. 145).

Partiendo y reconociendo una noción amplia de cuidado, ${ }^{2}$ la investigación en la que se basa este artículo ha conceptualizado al cuidado como la acción de ayudar a un niño o a una persona dependiente en el desarrollo y el bienestar de su vida cotidiana.

En esta definición, es central que las personas que reciban cuidados se encuentren en situación de dependencia. Con estos fines, las personas en situación de dependencia son aquellas que no pueden realizar las actividades de la vida diaria por sí mismas, y esto es debido a: la edad, en el caso de los/as niños/as y las personas mayores; y una discapacidad temporal o crónica, en el caso de las personas adultas.

Se reconocen las limitaciones que presenta acotar las situaciones donde se ejerce cuidado a aquellas donde existe 
${ }^{3}$ Esta definición fue inspirada en los aportes de Marie-Thérese LETABLIER (2001).

\footnotetext{
${ }^{4}$ Para un recorrido conceptual de los cuidados en la agenda de investigación y su vínculo con las políticas públicas, ver AGUIRRE et al., 2014.
}

algún grado de dependencia. Entre otras críticas, el cuidado así definido presenta la problemática de tener una concepción dualista y estática, en donde los que proveen cuidados son asumidos como totalmente autónomos e independientes y aquellos dependientes son lo opuesto. Por el contrario, el dar y recibir cuidados son actividades que en la realidad ocurren de forma simultánea, e incluso las personas consideradas autónomas reciben algún tipo de cuidado, por tanto se propone hablar de interdependencia (Valeria ESQUIVEL, 2012, p.150). A pesar de esta limitación en la definición que se elije, se reconoce que los cuidados son una necesidad de todas las personas durante toda la vida, aunque con diversas intensidades a lo largo del ciclo vital. La elección de acotar los cuidados a aquellos otorgados a quienes están en situación de dependencia responde a la necesidad de especificar el alcance de los cuidados en las investigaciones empíricas, así como al análisis de las políticas públicas (Rosario AGUIRRE et al., 2014, p. 49).

Desde esta perspectiva, el cuidado entonces implica hacerse cargo de los cuidados materiales, lo que conlleva un trabajo, de los cuidados económicos, que implican un costo económico, y de los cuidados psicológicos, que implican un vínculo afectivo, emotivo, sentimental. Los cuidados pueden ser realizados de manera honoraria o benéfica por parientes en el marco de la familia, o de manera remunerada en el marco o no de la familia. La naturaleza de estas tareas variarán según se realicen o no dentro de la familia, y también de acuerdo a s se trata o no de una tarea remunerada (AGUIRRE, 2004, p. 133, Karina, BATTHYÁNY, 2004, p.50). ${ }^{3}$ Esto significa que los cuidados no son por definición en sí mismos de carácter remunerado o no remunerado, sino como consecuencia de elecciones políticas, valoraciones culturales compartidas y sistemas de género.

La especificidad del trabajo de cuidado es la de estar basado en lo relacional, ya sea en el marco de la familia o por fuera de ella. El trabajo se realiza cara a cara entre dos personas, y genera lazos de proximidad en una situación de dependencia, pues una es tributaria de la otra para su bienestar y mantenimiento. De todas formas, lo que unifica la noción de cuidado es que se trata de una tarea realizada por mujeres, ya sea que se mantenga dentro de la familia, o que se exteriorice por la forma de prestación de servicios personales.

En los últimos años, los cuidados empiezan a adquirir protagonismo en la agenda de investigación, as como en las políticas públicas. ${ }^{4}$ El cuidado se distingue de otros tipos de trabajo no remunerado debido a su dimensión vincular, a partir de la cual está culturalmente asignado a las mujeres, 
como una responsabilidad 'naturalmente' femenina. Las características relacionales y afectivas de la tarea de cuidado están asociadas a la identidad femenina, lo que posiciona el cuidado como uno de los temas sustantivos directamente relacionados al real ejercicio de la ciudadanía social de las mujeres. Tomando el ejemplo de Arlie Russell HOCHSCHILD (2003), existe una imagen que simboliza el cuidado hasta la actualidad,

el retrato de una madre con su hijo en brazos. La madre que aparece allí suele estar en su casa sentada en un sillón, o en un escenario de ensueño, como un jardín. (...) quien brinda cuidado en ella no es un hombre, sino una mujer. No está en un lugar público, sino en su casa. Además, el cuidado que se retrata parece un acto natural, que no requiere esfuerzo. La mujer está sentada, quiescente, no parada ni en movimiento (posiciones asociadas con el "trabajo"). Parece disfrutar del cuidado que brinda a su hijo, y el rostro del hijo a menudo sugiere que la madre es buena en la tarea de cuidarlo (HOCHSCHILD, 2003, p.307).

La asociación tan estrecha y naturalizada de las mujeres con el cuidado termina por responsabilizarlas exclusivamente de la tarea, de sus costos, y genera obstáculos para su desarrollo en el ámbito público, y por tanto es clave para entender las desigualdades de género que persisten hasta la actualidad.

Paralelamente al desarrollo del concepto de cuidados, todavía en construcción, se elaboraron instrumentos metodológicos que permitieran operacionalizarlo y medir la distribución de este trabajo por sexo. Las Encuestas de Uso del Tiempo realizadas en el país en 2003, 2007 y 2013, son la herramienta que contribuye a visibilizar el trabajo no remunerado, entre los cuales el cuidado es uno de sus componentes claves.

La medición a través del uso del tiempo demostró que la carga global de trabajo de las mujeres (entendida como la suma del trabajo remunerado y no remunerado) es mayor que la de los varones, ya que las mismas se incorporan al mercado laboral sin renegociar su tiempo de trabajo en el hogar. Estas encuestas también permitieron visibilizar las vinculaciones entre trabajo remunerado y no remunerado, demostrando que las dificultades que tienen las mujeres en el acceso y permanencia en el trabajo remunerado son en parte debido a la carga en el trabajo no remunerado. En definitiva, evidenciaron que la distribución de tiempos y tareas está condicionada por la división sexual del trabajo.

Por otra parte, los esfuerzos de cuantificación y generación de indicadores buscan la valoración del trabajo 
${ }^{5}$ Producto de la importancia que adquieren los cuidados en el país, en el formulario 2013 de Encuesta de Uso del Tiempo se agregan preguntas específicas sobre cuidados, particularmente se indaga por cada población dependiente por separado y sobre contratación de cuidados externos al hogar. no remunerado, de manera a demostrar los costos económicos y sociales que conlleva. Contribuyen a visibilizar el trabajo realizado por las mujeres así como su aporte al bienestar al mostrar el rol que cumplen en el ámbito privado.

Finalmente, los datos que provienen de dichas encuestas constituyen una de las manifestaciones empíricas de la división sexual del trabajo, al mostrar las inequidades entre varones y mujeres en los cuidados de niños y personas dependientes. De esta manera contribuyen a visualizar la necesidad de que las políticas públicas planteen los cuidados como una responsabilidad social y colectiva y no como un problema individual a fin de disminuir las demostradas desigualdades de género.

Mientras que en un primer momento los cuidados eran parte de los distintos tipos de trabajo que estaban incluidos en el trabajo no remunerado medidos en dichas encuestas, en los últimos años se ha comenzado a concebir los cuidados en su especificidad, a entenderlos en relación con su contribución al bienestar y como actividad que trasciende el ámbito no remunerado. Por tanto se ha comenzado a buscar estrategias que permitan medirlos específicamente. ${ }^{5}$ Los hallazgos presentados en este artículo, derivados de la Encuesta Nacional de Cuidados No remunerados en Salud, pretenden contribuir en la línea desarrollada por las encuestas del uso del tiempo. Buscan profundizar en el análisis específico de los cuidados, pero particularmente en lo que a cuidados no remunerados se refiere y en un tipo de cuidado, los vinculados a la salud, poco explorados con anterioridad. Para dar cuenta de la especificidad del trabajo de cuidados no remunerados en salud, en este artículo se realizaran comparaciones con los datos provenientes de las Encuestas de Uso del Tiempo en los casos donde sea pertinente.

\section{Antecedentes de medición de los cuidados de salud no remunerados}

Si bien existe un recorrido considerable de las encuestas de uso del tiempo en la región latinoamericana y en Uruguay particularmente, persisten dimensiones que continúan sin ser suficientemente indagadas. Rosario AGUIRRE y Fernanda FERRARI $(2014$, p.16) sostienen que es necesario incorporar o mejorar la captación de las horas específicas de cuidado relevadas en dichas encuestas, otorgándole un tratamiento más exhaustivo y detallado. Asimismo presentan evidencias de que en las encuestas de uso del tiempo se presta mayor atención a la población infantil respecto a las personas mayores $\mathrm{y} / \mathrm{o}$ con discapacidad. Esta ausencia o escasez de información 
continúa siendo un obstáculo para dimensionar las necesidades de cuidado existentes, y sobre todo su posible redistribución.

En línea con estas reflexiones, la investigación que es insumo para este artículo pretendió, por un lado, profundizar en las horas de cuidado realizando una encuesta de uso del tiempo específica para su medición. Por otro lado, posibilitó dar cuenta de uno de los distintos tipos de cuidados, los cuidados no remunerados en salud, recibidos en mayor medida por personas mayores y por quienes tienen enfermedades crónicas.

La escasez de información y datos confiables sobre el papel que las familias cumplen en el cuidado de la salud tiene repercusiones negativas en la formulación y planificación de políticas de salud. Como indica Lourdes FERRÁN (2008, p.88), la omisión de indicadores sobre los servicios de salud domésticos impide que las políticas públicas puedan apreciar su efectivo valor en el marco de los sistemas de atención de salud. A partir de ello, es evidente la necesidad de nuevos indicadores que den cuenta del esfuerzo, medido en tiempos, que las familias realizan para contribuir al cuidado de la salud de sus miembros.

Los antecedentes regionales indican que, dadas las restricciones de recursos financieros que han enfrentado los servicios públicos de salud, son los hogares quienes deben enfrentar o complementar los cuidados de salud requeridos por un paciente, sea comprando los medicamentos, haciendo uso de los servicios privados o cuidando a los enfermos en el hogar (María Eugenia GÓMEZ LUNA, 2001 , p.2).

A nivel internacional, existen varios antecedentes de medición directa de los cuidados familiares en salud. Un antecedente a destacar es el de María del Mar GARCÍACALVENTE et al. (1999, p. 43) en España, quienes afirman que los cuidados no remunerados realizados por las familias son el principal proveedor de atención en salud, mientras que los servicios remunerados participan de forma secundaria. Estudios más recientes (DURÁN, 2008, p. 148) muestran que sólo el $12 \%$ del tiempo total dedicado anualmente al cuidado de la salud lo aportan los profesionales sanitarios, el $88 \%$ restante es tiempo dedicado por los familiares. La familia se constituye así en el principal prestador de cuidado a la salud. En el caso de Canadá, se estima que entre un $85 \%$ y un $90 \%$ del total de los cuidados prestados está por fuera del sistema de salud (NATIONAL COORDINATING GROUP ON HEALTH CARE REFORM AND WOMEN AND HOME CARE, 2002, p. 3).

Encuestas específicas realizadas en Canadá (HEALTH CANADA, 2004, p.14) buscaron establecer un perfil de los cuidadores familiares, determinar el tipo de hogar en que se 
realizan estos cuidados, qué tareas de cuidado específicas se realizan, en qué período de tiempo, y determinar los costos para quienes cuidan.

Dentro de los principales resultados de dicha encuesta se destaca que el $4 \%$ de los adultos canadienses están proporcionando actualmente cuidado familiar a otro que sufre de una discapacidad física o mental o enfermedad crónica. Esto se traduce en alrededor de un tercio de todos los tipos de cuidadores. La mayoría de dichos cuidadores (77\%) son mujeres y por lo general mayores de 45 años, las que constituyen el $51 \%$ de la población cuidadora del país. El $31 \%$ de los cuidadores son retirados y el $16 \%$ son amas de casa (HEALTH CANADA, 2004, p. 15).

Las mujeres, como grupo, están mucho más involucradas en el cuidado de las familias, mientras que los varones lo están en áreas tales como el pago de facturas, o llevar y traer personas enfermas. Una buena parte de los cuidados en salud en los hogares implica la atención a necesidades básicas como bañarse, alimentarse, entre otras, y en menor medida se realizan actos específicos como curar heridas dentro de los hogares (HEALTH CANADA, 2004, p.28).

En cuanto a las representaciones de los cuidadores en torno al cuidado, la mayoría los percibe como una responsabilidad de familia (67\%). Se destaca la existencia de un significativo grupo (35\%) que declara haber asumido este papel dado que no disponen de nadie más que se encargue del cuidado o debido a la falta de servicios externos de cuidado en el hogar (25\%) (HEALTH CANADA, 2004, p.26).

El estudio destaca la inversión de roles entre los hogares y el sistema institucional de salud, ya que al contrario de lo que suele pensarse, son los hogares los que apoyan al sistema sanitario. El uso de servicios formales parece ser exclusivo para aquellos miembros de la familia que requieren una atención intensiva.

En el caso español (GARCÍA-CALVENTE et al., 1999, p. 43), se puso de manifiesto que el $98 \%$ de quienes necesitan cuidados de salud los reciben de fuentes distintas a los servicios sanitarios institucionales, bien mediante el autocuidado (32\%), o mediante el sistema informal familiar (66\%), o ambos (1\%).

La investigación plantea que una de las consecuencias de las reformas de los sistemas sanitarios en España fue la derivación a los hogares de las responsabilidades y costos del cuidado de la salud. La reforma coloca el énfasis en la atención de la salud en el entorno del enfermo, transfiriendo al hogar más número de cuidados y de mayor complejidad (GARCÍA-CALVENTE et al., 2004, p.84).

Entre los estudios realizados es posible encontrar similitudes en el perfil de las personas cuidadoras: la mayoría 
son mujeres, personas mayores de 45 años y pertenecientes a niveles socio-económicos bajos con poco acceso al mercado de empleo (GARCÍA-CALVENTE et al., 1999, Daniel LA PARRA, 2001, GARCÍA-CALVENTE et al., 2004).

Los antecedentes constatan el rol fundamental de las mujeres en el sostenimiento (en base a trabajo invisible) del sistema de salud. En el caso español, las mujeres son el $60 \%$ de los cuidadores principales de personas mayores y el $75 \%$ en el caso de personas con discapacidad. Las mujeres asumen con mayor frecuencia el papel de cuidadoras secundarias, de tal manera que cuando una cuidadora principal necesita ayuda para cuidar, suele recurrir a otra mujer de la familia: madre, hermana o hija (GARCÍA- CALVENTE, 2004, p.84).

Como consecuencia de la sobre recarga de la actividad de cuidados no remunerados en salud, se expone a las mujeres a que su propia salud se vea afectada en algún grado, particularmente en la esfera psicológica. Los efectos también pueden apreciarse en el empeoramiento de sus posiciones en el mercado de empleo (ya sea por abandono temporal o definitivo del mismo o por la imposibilidad de acceder a él), en la situación económica personal, en la pérdida de redes sociales y en el disfrute del tiempo libre. Uno de los grandes costos que tiene el cuidado informal en salud para las mujeres es el escaso tiempo disponible (GARCÍA-CALVENTE et al., 2004, p.86).

A nivel regional, se destaca el antecedente de Chile (DíAZ et al., 2006, p.39), el que comparte todos los hallazgos identificados en las investigaciones europeas y canadienses, con la diferencia de que el tiempo dedicado al cuidado no difiere significativamente entre mujeres y varones.

Considerando estos antecedentes, puede señalarse que se cuenta con numerosa evidencia, centrada sobre todo en Europa, con la excepción de Chile en la región, respecto a que son las familias quienes realizan el mayor aporte en la provisión de cuidados en salud. La invisibilización de este aporte sustantivo para el bienestar social se asocia a la naturalización del trabajo no remunerado que ha sido llevado a cabo históricamente por las mujeres, sin un reconocimiento del mismo como tal.

Uruguay no contaba con antecedentes respecto a la medición del trabajo no remunerado en salud ni al perfil de las personas que lo proveen. Tampoco se contaba con información respecto a los costos derivados de la realización de estas tareas. Debido a la invisibilidad del aporte de las familias, no existen políticas específicas ni de coordinación entre el Sistema Nacional Integrado de Salud y otros organismos del Estado o la comunidad para apoyar a las familias en su rol de prestadoras de servicios no remunerados en salud. 
En cuanto a la organización del sistema de salud en Uruguay, cabe señalar que el país experimentó una reforma muy importante en los últimos años. Hasta el 2007 existían dos subsistemas que funcionaban en forma paralela. Un subsistema de salud pública, el que atendía a la población con menos recursos y otro privado, compuestos por Instituciones de Asistencia Médica Colectiva (IAMC) y seguros, que brindaba cobertura a través del pago de una cuota o de aportes de los/as trabajadores/as formales y sus empleadores/as (MINISTERIO DE SALUD PÚBLICA, 2010, p.67).

En 2007 se crea el Sistema Nacional Integrado de Salud que universaliza la atención en salud en el país. Entre las principales reformas, el nuevo Sistema integra el subsistema público y el privado, crea un seguro nacional de salud incluyendo en él a toda la población y no sólo a los trabajadores privados como en el anterior sistema. Universaliza el aseguramiento social incluyendo a los familiares. Transforma el modelo de atención avanzando en la implementación de la estrategia de Atención Primaria en Salud. Fortalece la política de Rectoría y Contralor y nivela la calidad y cantidad de las prestaciones. Por otra parte, incorpora la participación social como eje clave de la conducción de la reforma con la participación de usuarios y trabajadores (FERNÁNDEZ GALEANO y OLESKER (2009) en MINISTERIO DE SALUD PÚBLICA, 2010, p.68).

A pesar de estos avances que supusieron una mejora en el acceso a los servicios de salud de una importante proporción de la población, la población uruguaya presenta dificultades en el acceso a los cuidados en salud tanto en internación hospitalaria como en domicilio. El acceso está estratificado en función de los niveles socioeconómicos de los hogares y los lugares de residencia, ya que estos servicios no están distribuidos homogéneamente en el territorio. La cantidad de tiempo que sería necesario contratar a una persona durante un proceso de enfermedad significaría un costo muy alto para las familias de niveles socioeconómicos medios y bajos.

Junto a los servicios de hospitalización provenientes de instituciones públicas y privadas, en el país existen servicios privados (denominados servicios de acompañantes) que brindan cuidado cotidiano a enfermos durante la internación hospitalaria y en domicilio. Son servicios privados ofrecidos por el mercado que realizan tareas de cuidados en salud similares a las que realizan las familias de forma no remunerada. No existen servicios públicos gratuitos ni subsidiados similares.

En el caso de las personas que proveen cuidados remunerados de salud, quienes realizan tareas similares a las realizadas por los familiares, se observa que las mujeres 
- La Encuesta de Hogares Ampliada (2006) midió una gama más amplia de limitaciones en relación al censo. Fueron consideradas las limitaciones permanentes para utilizar los brazos o manos, para hablar y de limitaciones que le dificultan relacionarse con los demás. Para esta encuesta, de la población mayor de 65 años el $8,4 \%$ presenta limitaciones y un $5,6 \%$ de aquellos menores de 65 años. Del total de las mujeres el $9,1 \%$ de la población tiene limitaciones y del de hombres un $7,7 \%$. En general, las mujeres de más de 65 años presentan en mayor porcentaje limitaciones, respecto de los hombres en ese mismo tramo de edad. son la mayoría de los trabajadores del sector. Se trata de una población altamente feminizada y envejecida, probablemente como producto de las reglas del mercado laboral. Constituye un sector vulnerable en varios aspectos: la incidencia de la pobreza entre quienes trabajan en el sector es el doble de lo que ocurre para la población ocupada, la mayoría de los trabajadores no aporta a la Seguridad Social, su nivel educativo es bajo, la estabilidad en dichos puestos de trabajo está comprometida, los ingresos salariales son más bajos que el salario mínimo nacional, entre otras. Estas condiciones de trabajo dan cuenta de la carencia de valor social de la tarea de cuidados protagonizado por las mujeres. Por tanto, los cuidados cotidianos de salud, sean provistos por el mercado o por las familias, son realizados mayormente por mujeres, a partir de la división sexual del trabajo tradicional y en claras condiciones de vulnerabilidad asociadas a la invisibilización, la sobre-carga de trabajo, la informalidad, y la escasa valoración social de la tarea (AGUIRRE, 2013, p. 67). Dado el proceso de envejecimiento de la población uruguaya, el colectivo de personas mayores de 65 años se presenta como uno de los grandes demandantes de cuidados y particularmente de aquellos específicos de salud. La prevalencia de las enfermedades crónicas o de dependencia permanente es más alta en este grupo respecto a los colectivos de otras edades.

El alargamiento de la vida tiene como consecuencia la mayor duración del tiempo en que se necesitan cuidados. A medida que evoluciona el proceso de envejecimiento de la población, se incrementan los porcentajes de dificultades de visión, audición y movilidad prevalentes en la población, de acuerdo a los datos arrojados por el Censo 2011 . $^{6}$

Por ende, la disponibilidad de cuidadores es un aspecto fundamental para dar cobertura a dichas necesidades, la que encuentra limitantes en la rígida división sexual del trabajo que responsabiliza casi exclusivamente a las mujeres por estas tareas y a la negociación intergeneracional.

Respecto a la negociación inter-generacional, sobre cómo se distribuye el cuidado de las personas mayores dependientes, es claro que se encuentra mediada por los intereses y representaciones sobre los deberes y obligaciones de cuidado para cada generación. En base a los resultados de la Encuesta Nacional de Representaciones Sociales del Cuidado, Karina BATTHYÁNY, Natalia GENTA y Valentina PERROTTA (2012, p.55) identifican que existen diferentes concepciones sobre la situación deseada para el cuidado de las personas mayores. En este punto se evidencia una diferencia entre los menores de 69 años y los mayores de 70 años, ya que estos últimos perciben más fuertemente que 
los primeros la obligación de los/as hijos/as en el cuidado directo de padres y madres. Los datos muestran que hijos e hijas se sienten obligados a garantizar el cuidado, pero no necesariamente al cuidado directo. Esto da cuenta de una demanda insatisfecha en los cuidados familiares directos por parte de las personas mayores.

En los últimos años Uruguay ha avanzado en la ampliación de su sistema de protección social. Ejemplo de ello es el Plan de Equidad, el propio Sistema Nacional Integrado de Salud, la reforma en las asignaciones familiares y los niveles más altos de las jubilaciones y pensiones. En el marco de la discusión sobre el Sistema Nacional Integrado de Cuidados, la investigación de la que surge este documento aporta insumos para la caracterización y cuantificación del cuidado de salud no remunerado que brindan las familias y las mujeres y sus posibles articulaciones con el Sistema Nacional Integrado de Salud.

\section{Estrategia metodológica}

La investigación que dio origen a este artículo ubica a los cuidados en salud no solamente como una responsabilidad del sector sanitario, sino como un asunto que requiere del compromiso de diversos sectores del Estado, así como de la ciudadanía.

Para abordar los cuidados no remunerados en salud, se consideraron los desarrollos teóricos de la temática de cuidados desde la perspectiva de género. Los antecedentes de investigación relativos a los cuidados generan la necesidad de distinguir en su especificidad los cuidados en salud del resto de los cuidados cotidianos necesarios para el bienestar. En esta línea, en el proceso de operacionalización necesario para medir los cuidados no remunerados en salud se distinguieron, por un lado, aquellas actividades que se llevan a cabo en los hogares diariamente y que contribuyen a la prevención y promoción de salud en un sentido amplio (son las relevadas en las encuestas de uso del tiempo), y por otro lado, las tareas de cuidado no remunerado en salud.

En el primer caso, se encuentran las actividades destinadas a higienizar el hogar, preparar alimentos, higienizar a niños y niñas pequeños así como adultos dependientes, que sin duda implican actividades de trabajo no remunerado en salud ya que promueven el bienestar físico, psíquico y social de los individuos que habitan los hogares. Las encuestas de uso del tiempo muestran que el $30 \%$ de las mujeres en Uruguay dedican tiempo al cuidado infantil. En cuanto al cuidado de personas mayores y de personas dependientes, alrededor del $4 \%$ de la población 
uruguaya declara ser cuidadora para 2013 (BATTHYÁNY, 2015, p. 98).

Estos datos (y su respectiva medición en horas) están relevados en las encuestas de uso del tiempo y no forman parte del objeto de la Encuesta Nacional de Cuidados No Remunerados en Salud. Esta Encuesta se centró en el segundo tipo de tareas, aquellas de cuidado en salud destinadas a apoyar a las personas que tienen su capacidad de funcionamiento disminuida.

Se distinguen cuatro tipos de cuidados no remunerados en salud:

- Cuidados de salud específicos realizados en el hogar (hacer tratamientos, controlar manifestaciones de la enfermedad, administrar medicinas, etc.).

- Cuidados de apoyo a la salud realizados en el hogar ante situaciones de enfermedad (alimentar, higienizar, etc.).

- Acompañamiento (traslados específicos, entretener, concurrir a las consultas médicas, etc.);

- Gestiones con el sistema institucional de salud (solicitar consultas, retirar análisis, etc.).

La definición operativa del trabajo de cuidados no remunerados en salud construida para la Encuesta refiere a todas las tareas destinadas al cuidado en el ámbito de la salud realizadas para los miembros del hogar que no pueden hacerlas por sí mismas, sin recibir retribución monetaria por ellas. También incluyen los cuidados para personas que no viven en el hogar ya sean familiares o no.

Se consideran personas que no pueden realizar por sí mismas las actividades de cuidado de salud no remunerado: niños/as y niñas/as, personas mayores dependientes, personas con discapacidad inhabilitados para autocuidarse, enfermos crónicos, de episodios agudos inhabilitantes, terminales, etc.

La Encuesta se propuso describir y cuantificar los cuidados que proveen los hogares. Asimismo buscó conocer cómo se distribuye este trabajo entre varones y mujeres y cuáles son las consecuencias para la vida de éstos/as. Esta información aporta conocimiento fundamental sobre la contribución de las familias y de las mujeres al sistema sanitario, aportando datos sobre su magnitud en nuestro medio y enriqueciendo el concepto de cuidados que se ha vuelto clave en el marco de los sistemas de protección y bienestar.

En el presente artículo, se abordan algunas de las dimensiones indagadas por la Encuesta: 1) las horas de cuidado de salud no remunerado provistas por varones $y$ mujeres en las familias; 2) los tipos de tareas de cuidados en salud no remunerada que se proveen; 3) el perfil de las 


\footnotetext{
${ }^{7}$ La pregunta filtro para que un integrara la muestra era la siguiente: "Durante la última semana ¿usted o alguna persona del hogar ha realizado alguna tarea de cuidados por la que no ha recibido remuneración para una persona dependiente con enfermedad crónica o temporal o ha acompañado a alguna persona a una institución de atención en salud?"

${ }^{8}$ Tanto para los hogares en los que se realizaron cuidados no remunerados en salud como para los que no, se relevó información sobre las personas de carácter socio-demográfico. De esta forma, se obtuvieron datos sobre la composición de los hogares y sobre su nivel socioeconómico.
}

personas y de los hogares que brindan cuidados en salud no remunerado; y 4) la participación relativa de las familias en el cuidado de salud en comparación con las instituciones de salud.

Se trató de una encuesta representativa de la población uruguaya, basada en una muestra bietápica y estratificada por nivel socioeconómico, en la que se consultó a 1198 hogares sobre la realización de cuidados no remunerados en salud. Hasta el momento, esta es la primera encuesta que mide los cuidados no remunerados en salud, por tanto no se contaba con un marco muestral para definir un tamaño de muestra acorde con la distribución de los cuidados en la población. Debido a esto, se realizó un primer relevamiento para ubicar a los "hogares cuidadores", y luego un segundo relevamiento en donde se pudo medir efectivamente los cuidados que se brindan. Los antecedentes de investigación (GARCÍA-CALVENTE et al., 1999, DÍAZ et al., 2006) evidenciaban que entre $10 \%$ y $20 \%$ de los hogares brindan cuidados no remunerados en salud, y dichas investigaciones plantearon estrategias de abordaje similares a la mencionada para Uruguay.

En el primer relevamiento, se detectaron de manera aleatoria 210 hogares en los que se realizaron cuidados no remunerados en salud.?

Ese total fue aumentado a 300 , con el objetivo de ser analizado específicamente en base a una muestra confiable. ${ }^{8}$

Respecto a las ventajas y desventajas asociadas a la aplicación de esta técnica, se destaca como ventaja el esfuerzo por visibilizar el cuidado a través del tiempo de dedicación, el cual continúa siendo actualmente un desafío que apunta a la transformación de las relaciones de género. Esto se aplica especialmente para la región latinoamericana en la que, a pesar de los importantes avances en la materia, no se cuenta aún con encuestas de uso de tiempo periódicas y comparables entre los países de la región. En definitiva, esta investigación permite aportar elementos para la comprensión del cuidado como un trabajo no remunerado, medible en horas, sin desconocer que es esta una de las tantas y complementarias líneas de análisis para su comprensión.

Como desventaja ha de señalarse que el tiempo es subjetivo y no es lineal, englobando una serie de subjetividades y sucesos que son incompresibles desde una concepción cronológica del mismo. Se ha priorizado el hecho de que al mismo tiempo el tiempo es un recurso utilizado y distribuido de forma desigual entre mujeres y varones, lo que hace legítimo su análisis.

A continuación se presenta el análisis de algunos de los resultados de la Encuesta de Cuidados No Remunerados en Salud. 


\section{Principales hallazgos}

\section{1 ¿Quiénes brindan cuidados de salud no remunerados?}

Este apartado profundiza en el perfil de las personas y los tipos de familias que realizan cuidados no remunerados en salud. En Uruguay, el 17,5\% de los hogares brindan cuidados no remunerados en salud.

En cuanto a la tipología de hogares, los que brindan cuidados en salud presentan una proporción mayor de hogares biparentales con hijos $(37,7 \%)$, extensos $(14,3 \%)$ y compuestos (15,3\%), en comparación con los que no lo hacen (cuyas cifras son $30,0 \%, 4,4 \%$ y $8,9 \%$ respectivamente). Estos tres tipos de hogares son más numerosos y por tanto cuentan con mayor número de potenciales cuidadores y beneficiarios del cuidado en salud. Como se analizará más adelante, aproximadamente el $60 \%$ de los cuidados se realizan entre miembros del hogar.

Cuadro 1 - Tipo de hogar según provisión de cuidados en salud

\begin{tabular}{|l|l|l|l|}
\hline & $\begin{array}{l}\text { Hogares } \\
\text { cuidadores }\end{array}$ & $\begin{array}{l}\text { Hogares } \\
\text { no } \\
\text { cuidadores }\end{array}$ & Total \\
\hline Unipersonal & 8,3 & 22,3 & 19,0 \\
\hline $\begin{array}{l}\text { Biparental sin } \\
\text { hijos }\end{array}$ & 17 & 23,6 & 22,0 \\
\hline $\begin{array}{l}\text { Biparental } \\
\text { con hijos }\end{array}$ & 37,7 & 30 & 31,8 \\
\hline $\begin{array}{l}\text { Monoparental } \\
\text { femenino }\end{array}$ & 6 & 8,6 & 8,0 \\
\hline $\begin{array}{l}\text { Monoparental } \\
\text { masculino }\end{array}$ & 0,3 & 1,1 & 0,9 \\
\hline Extenso & 14,3 & 4,4 & 6,7 \\
\hline Compuesto & 15,3 & 8,9 & 10,4 \\
\hline Sin núcleo & 1 & 1,2 & 1,2 \\
\hline Total & 100 & 100 & 100 \\
\hline
\end{tabular}

Fuente: Elaboración propia en base a la Encuesta Nacional de Cuidados No Remunerados en Salud. Uruguay 2013 
Respecto a los cuidadores/as, se observa el fuerte protagonismo de las mujeres, quienes representan el 76,6\% de quienes brindan cuidados en salud no remunerados. Los datos concuerdan con los de la Encuesta de Uso del Tiempo 2013, que muestran que las mujeres participan en el cuidado infantil (niños/as de 0 a 12 años) en el $80,0 \%$ de los casos mientras que lo hacen el $59,2 \%$ de los varones. En el caso de las personas con discapacidad estas cifras con de $79,9 \%$ y de $49,7 \%$ respectivamente.

Los cuidados no remunerados en salud se brindan en su gran mayoría a familiares $(92,2 \%)$, ya sea que vivan dentro $(58,0 \%)$ o fuera del hogar $(34,2 \%)$. Se observan diferencias importantes entre varones y mujeres cuidadores/ as. El $73,9 \%$ de los cuidados que realizan los varones son a miembros del hogar. Sin embargo, las mujeres no cuidan de forma tan concentradamente a miembros del hogar, sino que el $45,4 \%$ de sus cuidados están destinados a personas fuera del hogar. Por tanto, se evidencia que las mujeres brindan ayuda a otros hogares en mayor medida que los varones, convirtiéndose en un eslabón central en la estrategia de cuidado con la que cuentan los hogares.

Cuadro 2 - Tipo de vínculo entre beneficiario/a y cuidador/a por sexo del cuidador/a

\begin{tabular}{|l|l|l|l|}
\hline & Varones & Mujeres & Total \\
\hline Parientes que integran el hogar & 70,8 & 53,9 & 58,0 \\
\hline Parientes fuera del hogar & 18,8 & 39,1 & 34,2 \\
\hline No parientes fuera del hogar & 7,3 & 6,3 & 6,5 \\
\hline No parientes que integran el hogar & 3,1 & 0,7 & 1,2 \\
\hline Total & 100 & 100 & 100 \\
\hline
\end{tabular}

Fuente: Elaboración propia en base a la Encuesta Nacional de Cuidados No Remunerados en Salud. Uruguay 2013

El cuadro 3 muestra la edad de los beneficiarios del cuidado según el sexo de quien los cuida. Se observa que, de los cuidados realizados por mujeres, el 43,7\% está destinado a personas mayores de 70 años, mientras que esto ocurre con solo el $30,3 \%$ del cuidado que realizan los varones. 
Cuadro 3 - Edad en tramos de los y las beneficiarias/os del cuidado por sexo del cuidador/a

\begin{tabular}{|l|l|l|l|}
\hline & Varones & Mujeres & Total \\
\hline $\begin{array}{l}\text { Menores de 4 } \\
\text { afios }\end{array}$ & 2,1 & 4,6 & 4,0 \\
\hline Entre 4 y 12 afios & 17,7 & 12,2 & 13,5 \\
\hline $\begin{array}{l}\text { Entre 13 y 69 } \\
\text { años }\end{array}$ & 50,0 & 39,5 & 42,0 \\
\hline $\begin{array}{l}\text { Entre 70 y } 79 \\
\text { afios }\end{array}$ & 11,5 & 16,1 & 15,5 \\
\hline Desde 80 afios & 18,8 & 27,6 & 25,5 \\
\hline Total & 100 & 100 & 100 \\
\hline
\end{tabular}

Fuente: Elaboración propia en base a la Encuesta Nacional de Cuidados No Remunerados en Salud. Uruguay 2013

Si bien en todos los niveles socioeconómicos son las mujeres las que cuidan en mayor proporción que los varones, esta diferencia se incrementa en el caso del nivel socioeconómico bajo. En este nivel los varones se involucran menos en los cuidados en salud no remunerados que las mujeres: el $14 \%$ de los cuidadores en salud no remunerados de nivel bajo son varones, mientras que son el $26 \%$ de los cuidadores de nivel medio y el $21 \%$ de los de nivel alto, lo que coincide con la tendencia encontrada respecto a las Encuestas del Uso del Tiempo, así como a las representaciones sociales del cuidado (BATTHYÁNY, GENTA, PERROTTA, 2012, p.21).

Los datos de EUT 2013 muestran que la carga de trabajo que las mujeres realizan en materia de cuidado a personas dependientes aumenta a medida que disminuyen los ingresos de las personas. Mientras que la tasa de participación de las mujeres que residen en hogares pertenecientes al primer quintil de ingresos es de $60,0 \%$, en aquellas del quinto quintil este valor desciende a $12,5 \%$. Las brechas de género, por su parte, aumentan a medida que disminuyen los ingresos de las personas (BATTHYÁNY, GENTA, PERROTTA, 2015, p.60).

Por otra parte, los datos de la Encuesta Nacional de Representaciones Sociales del Cuidado evidencian que en 
los sectores de bajos ingresos, en mayor medida que en los otros niveles, persisten valoraciones más familistas y que tienden a cargar la responsabilidad del cuidado en las mujeres (BATTHYÁNY, GENTA, PERROTTA, 2012, p.28).

En esta línea, al observar el vínculo que los cuidadores en salud no remunerados tienen con el trabajo remunerado, encontramos que si bien las mujeres cuidadoras presentan menor participación en el mercado laboral que los varones cuidadores, son las mujeres de nivel socioeconómico bajo las que se encuentran en una situación más vulnerable respecto a contar con ingresos propios. Así, tan solo el $27 \%$ de las mujeres cuidadoras no remuneradas en salud acceden al trabajo remunerado, mientras esto ocurre con el $50 \%$ de los varones cuidadores del mismo nivel.

La encuesta evidencia por tanto un grupo de mujeres que se dedica fuertemente a las tareas de cuidados no remunerados y no accede al trabajo remunerado, con los consecuentes impactos en su autonomía económica.

Gráfico 1 - Proporción de cuidadores/as no remunerados/as en salud que tienen trabajo remunerado por sexo y nivel socioeconómico.

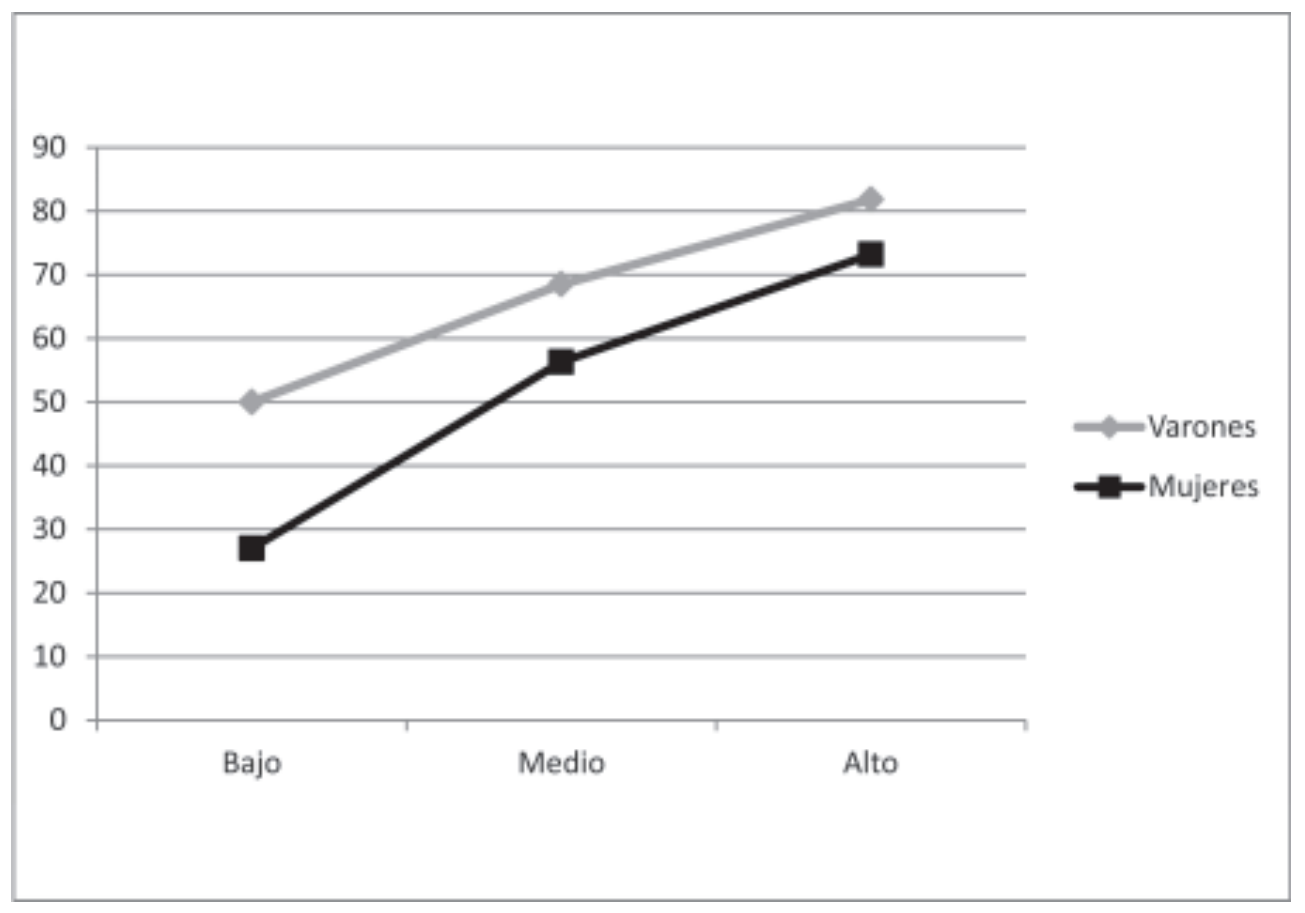

Fuente: Elaboración propia en base a la Encuesta Nacional de Cuidados No Remunerados en Salud. Uruguay 2013 
En la actualidad, las mujeres continúan presentando mayores dificultades que los varones para articular trabajo remunerado y cuidados. Los datos para Uruguay muestran un comportamiento opuesto entre varones y mujeres respecto a la participación en el mercado laboral cuando tienen hijos menores, dado que mientras los varones incrementan su participación, las mujeres la reducen. Este indicador evidencia el impacto que tiene el cuidado en las trayectorias laborales de las mujeres.

Respecto al perfil de quienes brindan cuidados no remunerado en salud, observamos que es el tramo entre 46 y 65 años el que presenta la mayor proporción de personas que brindan dichos cuidados (44\%). Probablemente esto esté asociado a que en este tramo etario conviven personas que cuidan tanto a sus padres y madres adultos/as mayores como a sus nietos/as. En el caso de las mujeres, esta proporción es más relevante que en el caso de los varones ( $47 \%$ vs. $37 \%$ ), concentrándose aproximadamente la mitad de las mismas en este tramo etario.

Se evidencia también la baja participación de los jóvenes en estas tareas en comparación con las personas adultas y adultas mayores. Solo el $11 \%$ de los cuidadores/ as tiene menos de 30 años. Si comparamos estos datos con los resultados de la Encuesta de Uso del Tiempo realizada en 2007 (INSTITUTO NACIONAL DE ESTADÍSTICA, 2008, p. 24), se observa que el tramo etario dónde se concentraba la mayor proporción de personas que realizaban trabajo no remunerado era el de 30 a 45 años, edad asociada a la mayor demanda de cuidados de niños/as.

Los datos presentados indican que los cuidados no remunerados en salud son brindados por personas de mayor edad. Esto podría explicarse tanto por la simultaneidad de personas dependientes que cuidan (niños/as y personas mayores), como por las mayores posibilidades que tienen las personas mayores de cubrir el cuidado ante situaciones extraordinarias, que desbordan la estrategia de cuidado cotidiana (enfermedades temporales, accidentes) debido a pertenecer en mayor medida a la población inactiva.

\section{2 ¿Cuántas horas dedican mujeres y varones al cuidado de salud no remunerados?}

El promedio de horas semanales que la población menor de 65 años destina en Uruguay al cuidado no remunerado en salud es de 53 horas, siendo de 56 horas para las mujeres y 44 para los varones. Se observa un mayor número de horas de dedicación al cuidado no remunerado de salud respecto a los cuidados cotidianos (Encuestas de 
${ }^{9}$ Por ejemplo, las horas destinadas al acompañamiento de enfermos durante la internación hospitalaria, o las horas requeridas de cuidado infantil mientras dura un proceso de enfermedad como la gripe.
Uso del Tiempo, 2003, 2007 y 2013). En la EUT 2013 surge que el promedio de horas semanales que las mujeres dedican al cuidado es de 22 horas, mientras que los varones dedican 17 horas (BATTHYÁNY, 2015, p. 98).

Dicha diferencia probablemente se explique porque el cuidado de salud requiere un cuidado más intensivo durante el tiempo que se desarrolla una enfermedad, ${ }^{9}$ a excepción de casos de personas con dependencia crónica, las cuales tienen mayores requerimientos de cuidados en forma permanente.

Al analizar la cantidad de horas semanales de cuidado que destinan los cuidadores/as en función de su edad, se observa que las mujeres dedican más horas al cuidado en salud no remunerado en todos los tramos etarios, con excepción de las personas mayores de 65 años. En este caso, ocurre el fenómeno contrario, ya que los varones cuidan mayor número de horas que las mujeres. Al indagar con mayor detenimiento a estos varones, se pueden señalar algunas tendencias que necesitan mayor profundización dado el escaso número de varones que cuidan.

En general, podemos decir que estos varones son personas mayores que no trabajan de forma remunerada, que cuidan a sus cónyuges también mayores y en la mayoría de los casos con dependencia crónica, y que conforman hogares biparentales sin hijos. Por tanto, su mayor dedicación horaria puede deberse a que conviven con la demanda cotidiana y continua de sus cónyuges mayores enfermas. Otra hipótesis posible es que dichos varones tienen mayor dedicación al cuidado porque no se enfrentan (por el momento del ciclo vital) a las obligaciones propias del mercado laboral o lo hacen en menor medida que en otras etapas de la vida, con lo que se encuentran más disponibles para el cuidado. La reducción de las responsabilidades que caracterizan el rol del varón proveedor a estas edades, puede producir un "relajamiento" que deje a los varones más disponibles para el cuidado. Por otro lado, la escasez de apoyos de los hijos y sobre todo hijas que tienen menor disponibilidad de tiempo que antes para brindar cuidado, también puede presionar para que los varones conyugues asuman en mayor medida las tareas de cuidado.

La estrategia utilizada por los hogares para cubrir la demanda de cuidados en salud incluye los cuidados brindados por sus mismos integrantes, pero también apoyos externos como: contratación de personas, trabajo no remunerado de parientes y no parientes y servicios brindados por instituciones públicas y privadas. El $18 \%$ de los hogares que brindan cuidados no remunerados en salud recibe apoyo externo para el cuidado de la salud de sus miembros. 
Gráfico 2 - Promedio de horas semanales dedicadas al cuidado no remunerado en salud según sexo y edad del cuidador/a.

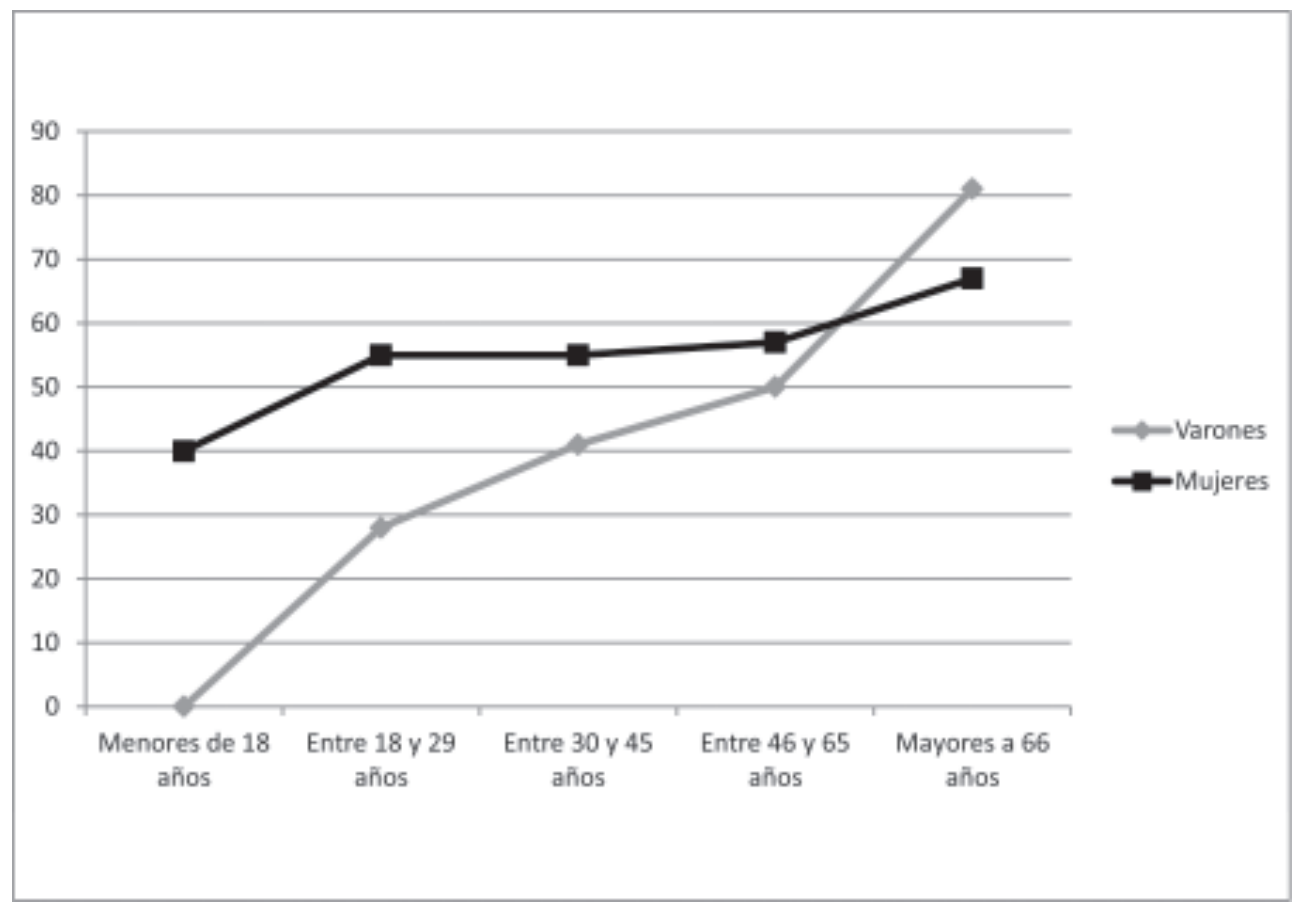

Fuente: Elaboración propia en base a la Encuesta Nacional de Cuidados No Remunerados en Salud. Uruguay 2013

Al analizar el promedio de horas semanales de cuidado que recibe el hogar desde fuera (remuneradas y no remuneradas) se observa que es muy inferior al promedio de horas semanales que brindan sus propios miembros (79 vs. 31).

Por otra parte, cuando se analiza la distribución de las horas de cuidado en función de su carácter remunerado y no remunerado, se evidencia que la mayor parte de las horas recibidas por el hogar son no remuneradas, ya sean brindadas por sus propios integrantes como por personas externas al hogar (90 vs. 23). Los hogares que cuentan con cuidados externos reciben un promedio de 23 horas semanales de cuidado remunerado en salud provenientes de servicios (de acompañantes de enfermos/as o adultos/ as, centros infantiles donde se realizan tratamientos médicos, centros de larga estadía, centros de día) y de personas contratadas. 


\section{Reflexiones finales}

Los datos presentados en este artículo evidencian en primer lugar que las familias dedican un mayor número de horas al cuidado no remunerado de salud cuando lo comparamos con los cuidados cotidianos. El cuidado de salud, por tanto, requiere una dedicación más intensiva durante el tiempo que se desarrolla una enfermedad tanto sea crónica como temporal.

En segundo lugar, el cuidado en salud es mayormente realizado por integrantes del hogar, y de forma no remunerada. Solo $18 \%$ de los hogares encuestados han utilizado algún tipo de cuidado externo (remunerado y no remunerado) para cubrir la necesidad de cuidados por enfermedad de sus miembros.

La cantidad de tiempo que sería necesario contratar a una persona durante un proceso de enfermedad significaría un costo muy alto para las familias de niveles socioeconómicos medios y bajos. Las dificultades en el acceso conducen, dada la división sexual del trabajo imperante, a una mayor dedicación de las mujeres de las familias a estos cuidados de forma no remunerada.

En tercer lugar, las mujeres son las principales cuidadoras en salud (76,6\%), lo que da cuenta de la división sexual del trabajo existente en estas tareas. Por su parte, son las mujeres de mayor edad las que se dedican principalmente a estas tareas, y esto es un rasgo distintivo de estos cuidados específicos que los diferencia de los cuidados no remunerados cotidianos. Esto probablemente se deba a la simultaneidad de personas dependientes que cuidan (niños/as y personas mayores) y a las mayores posibilidades que tiene esta población de cubrir el cuidado ante situaciones extraordinarias, que desbordan la estrategia de cuidado cotidiana (enfermedades temporales, accidentes).

También puede observarse que en relación al tipo de cuidado que brindan las mujeres, éste parece ser más exigente que el que brindan los varones, dado que, en primer lugar, éstas realizan cuidados en mayor medida que los varones para otros hogares, con los costos que trae el hecho de trasladarse en términos de tiempo, cansancio, etc. En segundo lugar, es más exigente desde el punto de vista de las personas beneficiarias, ya que a diferencia de los varones, las mujeres cuidan en mayor medida a personas mayores respecto a niños/as. La mayor carga de cuidado de salud a personas mayores tiene altos niveles de exigencia desde el punto de vista de quien cuida (BATTHYÁNY, GENTA, PERROTTA, 2013, p.31). ${ }^{10}$

Por tanto, la división sexual del trabajo en este caso cuidado a adultos/as mayores.

\begin{abstract}
no sólo se observa en la mayor participación de las mujeres
\end{abstract}


y en la mayor cantidad de horas dedicadas (12 horas de diferencia), sino en el tipo de cuidado brindado. Podría afirmarse entonces que hay una división sexual cuantitativa y cualitativa del trabajo de cuidado de salud brindado en los hogares. Este hallazgo es análogo al encontrado en las investigaciones sobre uso del tiempo en trabajo no remunerado (BATTHYÁNY, 2009, p. 108).

Esta división sexual del trabajo tiene consecuencias en el ejercicio de derechos de las mujeres, entre ellos el acceso al trabajo remunerado. Así, tan solo el $27 \%$ de las mujeres cuidadoras del nivel socioeconómico bajo acceden al trabajo remunerado, lo que evidencia las dificultades que presenta la carga de cuidados de salud no remunerado para la autonomía económica de las mujeres. Son las mujeres de mayores niveles socioeconómicos las que tienen mayor disponibilidad económica para costear servicios de cuidado externo durante el tiempo que dure la enfermedad de sus familiares.

Por tanto, los cuidados cotidianos de salud son realizados mayormente por mujeres, a partir de la división sexual del trabajo tradicional, y en claras condiciones de vulnerabilidad asociadas a la invisibilización, la sobrecarga de trabajo y la escasa valoración social de la tarea.

En el marco de la construcción del Sistema Nacional Integrado de Cuidados, la alta provisión de cuidados no remunerados en salud que proveen los hogares y las mujeres al sistema de salud habilitan a reflexionar sobre el grado de institucionalización de la salud a la que se aspira, a sabiendas de que la poca o nula institucionalización, con sus discutidas consecuencias, tiene costos para el ejercicio de derechos de quienes se hacen cargo de dichas tareas, las mujeres. Cabe preguntarse cuál es el rol deseado del sistema institucional de salud en la provisión de cuidados frente a enfermedades temporales, accidentes y enfermedades crónicas.

Finalmente, a partir de estos hallazgos, cabe plantearse algunas interrogantes a futuro: ¿Cómo es posible incluir los cuidados específicos en salud en el sistema de prestaciones de cuidados? ¿Cuál es el tipo de complementariedad posible entre el Sistema Nacional Integrado de Cuidados y el Sistema de Salud? ¿Qué medidas son necesarias para valorizar la tarea de cuidados, sea esta desarrollada dentro del hogar o en el mercado? ¿Cómo generar acciones que valoricen la tarea de cuidados en el mercado sin sumar excesivos costos a las familias quienes contratan actualmente estos servicios? ¿Cómo se resuelve la cobertura de las necesidades de cuidado de una forma que no sobre recargue a las mujeres? 


\section{Bibliografía}

AGUIRRE, Rosario y FERRARI, Fernanda. La construcción del sistema de cuidados en el Uruguay. En busca de consensos para una protección social más igualitaria. Santiago de Chile: Editora CEPAL- Cooperación Alemana. Serie Políticas Sociales, Número 193, 2014.

AGUIRRE, Rosario, BATTHYÁNY, Karina, GENTA, Natalia y PERROTTA, Valentina. "Los cuidados en la agenda de investigación y en las políticas públicas en Uruguay". Quito: Editora Íconos. Revista de Ciencias Sociales. Número 50, 2014, p. 43-60.

AGUIRRE, Rosario. Personas Ocupadas en el Sector Cuidados. Montevideo: Editora Sistema Nacional de Cuidados, 2013.

AGUIRRE, Rosario. "Trabajo no remunerado y uso del tiempo. Fundamentos conceptuales y avances empíricos. La encuesta Montevideo 2003. En: Enrique MAZZEl (Comp.) El Uruguay desde la Sociología II. Montevideo: Editora Departamento de Sociología-FCS-UdelaR, 2004. p. 127152.

BATTHYÁNY, Karina. "Los tiempos del cuidado en Uruguay, En: BATTHYÁNY, Karina (Coord.) Los tiempos del bienestar social. Montevideo: Editora Doble Clic. UNFPA. ONU Mujeres. INMUJERES. FCS Udelar, 2015, p. 87-134.

BATTHYÁNY, Karina, GENTA, Natalia y PERROTTA, Valentina. Los cuidados de calidad desde el saber experto y su impacto de género. Análisis comparativo sobre cuidado infantil $y$ de adultos y adultas mayores en el Uruguay. Santiago de Chile: Editora CEPAL, Serie Asuntos de Género. Número 123, 2013.

BATTHYÁNY, Karina, GENTA, Natalia y PERROTTA, Valentina. La población uruguaya y el cuidado: Persistencias de un mandato de género Encuesta nacional sobre representaciones sociales del cuidado: Principales resultados. Santiago de Chile: Editora CEPAL, Serie Asuntos de Género. Número 117, 2012.

BATTHYÁNY, Karina. "Cuidado de personas dependientes y género. En: AGUIRRE, Rosario (Ed.) Las bases invisibles del bienestar social. El trabajo no remunerado en Uruguay. Montevideo, Editora Doble clic-UNIFEM, 2009, p. 87-124

BATTHYÁNY, Karina. Cuidado infantil y trabajo: ¿un desafío exclusivamente femenino? Una mirada desde el género y la ciudadanía social, Montevideo: Editora CINTERFOR/ OIT, 2004.

DÍAZ, Ximena; MAURO, Amalia y MEDEL, Julia. Cuidadoras de la vida: Visibilización de los costos de la producción de salud en el hogar. Impacto sobre el trabajo total de 
las mujeres. Santiago de Chile: Editora Centro de Estudios de la Mujer, 2006.

DURÁN, María Ángeles. "Diez buenas razones para medir el trabajo no remunerado en el cuidado de la salud. En: ORGANIZACIÓN PANAMERICANA DE LA SALUD, La economía invisible y las desigualdades de género. La importancia de medir y valorar el trabajo no remunerado, Washington, D.C.: Editora OPS, 2008, p. 147148.

ESQUIVEL, Valeria. "Cuidado, economía y agendas políticas: una mirada conceptual sobre la organización social del cuidado en América Latina. En: ESQUIVEL, Valeria (Ed.) La economía feminista desde América Latina: Una hoja de ruta sobre los debates actuales en la región. Santo Domingo: Editora ONU-Mujeres, 2012.

FERRÁN, Lourdes. Marco conceptual y lineamientos metodológicos de la cuenta satélite de los hogares para medir el trabajo no remunerado en salud. En: ORGANIZACIÓN PANAMERICANA DE LA SALUD. La economía invisible y las desigualdades de género. La importancia de medir y valorar el trabajo no remunerado. Washington, D.C.: Editora OPS, 2008. p. 8798.

GARCÍA-CALVENTE, María del Mar; MATEO, Inmaculada y GUTIERREZ, Pilar. Cuidados y cuidadores en el sistema informal de salud. Granada: Editora Escuela andaluza de salud pública e Instituto Andaluz de la Mujer, 1999.

GARCÍA-CALVENTE, María del Mar; MATEO, Inmaculada y MAROTO, Gracia. El impacto de cuidar en la salud y la calidad de vida de las mujeres. Barcelona: Editora Revista Gaceta Sanitaria Volumen 18, Número 5, 2004.

GÓMEZ LUNA, María Eugenia. Cuenta satélite de los hogares: valoración del trabajo doméstico no pagado. El caso de México. México: Editora INEGI, 2001.

HEALTH CANADA. Informal/Family Caregivers in Canada Caring for Someone with a Mental Illness, Toronto: Editora Decima Research Inc. 2004.

HOCHSCHILD, Arlie. The Commercialization of Intimate Life: Notes from Home and Work. Berkeley: Editora University of California Press, 2003.

INSTITUTO NACIONAL DE ESTADÍSTICA. Uso tiempo del Tiempo y trabajo no remunerado en el Uruguay. Módulo de la Encuesta Continua de Hogares Septiembre 2007. Montevideo: Editora INSTITUTO NACIONAL DE ESTADÍSTICA, 2008.

LA PARRA, Daniel. Contribución de las mujeres y los hogares más pobres a la producción de cuidados de salud informales. Alicante: Editora Revista Gaceta Sanitaria, Volumen 15 Número 6, 2001. 
LETABLIER, Marie-Thérese. Le travail centr sur autrui e sa conceptualization en Europe. Paris: Editora Revista Travail, Genre et Societés, Número 6, 2001.

MINISTERIO DE SALUD PÚBLICA. La construcción del Sistema Nacional Integrado de Salud 2005-2009. Montevideo: Editora MSP, 2010.

MONTAÑO, Sonia. El cuidado en Acción. Santiago de Chile: Editora CEPAL-UNIFEM, Serie Cuadernos de la CEPAL Número 94, 2010.

NATIONAL COORDINATING GROUP ON HEALTH CARE REFORM AND WOMEN AND HOME CARE. Women and home care. Why does home care matter to women? Toronto: Editora York University, 2002.

THOMAS, Carol. "Deconstruyendo los conceptos de cuidados. En: CARRASCO, Cristina, BORDERÍAS, Cristina y TORNS, Teresa (Eds.). El trabajo de cuidados: historia, teoría y políticas. Madrid: Editora Catarata, 2011 , p. 145176.

[Recebido em 13/01/2015,

reapresentado em 06/05/2016

e aceito para publicação em 03/07/2016]

The contribution of family and women to unpaid health care in Uruguay

Abstract: Considering the growing importance of care in public agenda and research in Uruguay, this paper focuses on one type of unpaid care that requires more visualization: unpaid health care. It aims to answer the following questions: Who bears the costs of unpaid health care in Uruguay? What is the profile of unpaid caregivers in health care? What relationship do they have with the institutional health system? It uses information from the National Survey of Unpaid Health Care, conducted by the Research Group Sociology of Gender, of which the authors of this article are members. The survey is representative of the Uruguayan population, based on a two-stage sample stratified by socioeconomic level, where 1,198 homes were consulted about unpaid health. It demonstrates that health care is mostly done by household members, on unpaid basis. Women are the main caregivers in health, what shows the existing sexual division of labor in these tasks. In turn, it is older women who are mostly dedicated to such care, and this is a particular feature that differentiates the health care of unpaid everyday care.

Keywords: Gender; Care, Health; Time Use; Unpaid Work

\begin{abstract}
A contribuição das famílias e mulheres aos cuidados não remunerados com a saúde no Uruguai

Resumo: Considerando o crescente protagonismo dos cuidados na agenda pública y de pesquisa no Uruguai, o presente artigo está focado em um tipo de cuidado prestado de forma não remunerada que requer mais atenção: o cuidado não remunerado em saúde. Este estudo se propõe a responder às seguintes questões: Quem se responsabiliza pelos custos do cuidado não remunerado em saúde no Uruguai? Qual é o perfil das pessoas cuidadoras não remuneradas em saúde? A quem cuidam? Que vínculo mantém com o sistema institucional de saúde? Para tal propósito, a investigação usa informação proveniente da Encuesta Nacional de Cuidados No Remunerados en Salud (Questionário Nacional de Cuidados Não Remunerados em Saúde), realizada pelo Grupo de Investigación Sociología de Género, do qual as autoras são integrantes. Tal questionário, representativo da população uruguaia, está baseado em uma amostra de duas etapas e estratificado por nível socioeconómico, em que foram consultados 1.198 lares
\end{abstract}


sobre a realização de cuidados não remunerados em saúde. Se demonstra que o cuidado em saúde é realizado principalmente por integrantes do lar, e de forma não remunerada. As mulheres são as principais cuidadoras em saúde, o que mostra a divisão sexual do trabalho existente nestas tarefas. Por sua vez, são as mulheres de mais idade que se dedicam principalmente a esses cuidados, e esta é uma característica particular que diferencia os cuidados com a saúde dos cuidados não remunerados cotidianos.

Palavras-chave: Gênero, cuidado, saúde, uso do tempo, trabalho não remunerado

Karina Batthyány (karina.batthyany@cienciassociales.edu.uy). Doctora en Sociología por la Univeriste de Versailles Saint Quentin en Yvelines, Francia. Profesora Agregada Grado 4. Facultad de Ciencias Sociales. Universidad de la República. Líneas de investigación: Género, cuidados y responsabilidades familiares, uso del tiempo, metodología de la investigación.

Natalia Genta (natalia.genta@cienciassociales.edu.uy). Candidata a Doctora en Sociología por Facultad de Ciencias Sociales, Universidad de la República, Uruguay. Máster en Ciencias Sociales con mención en Género y Desarrollo por FLACSO Ecuador. Asistente Grado 2 Departamento de Sociología, Facultad de Ciencias Sociales. Universidad de la República. Líneas de investigación: Género, cuidados, migraciones.

Valentina Perrotta (valentina.perrotta@cienciassociales.edu.uy). Magister en Género, Sociedad y Políticas (PRIGEPP-FLACSO). Estudiante de Doctorado por Facultad de Ciencias Sociales, Universidad de la República, Uruguay. Ayudante Grado 1, Departamento de Sociología, Facultad de Ciencias Sociales Universidad de la República. Líneas de investigación: Género, cuidados, políticas públicas. 